\title{
Photochemistry in synthesis: Where, when, and why*
}

\author{
Stefano Protti ${ }^{1}$, Daniele Dondi ${ }^{1,2}$, Maurizio Fagnoni ${ }^{1}$, and \\ Angelo Albini ${ }^{1, \neq}$
}

${ }^{1}$ Department of Organic Chemistry, University of Pavia, via Taramelli 10, 27100 Pavia, Italy; ${ }^{2}$ INCA Consortium, Via delle Industrie, 21/8-30175 Marghera (VE), Italy

\begin{abstract}
A series of photochemical reactions are assessed under the environmental aspect by using Eissen and Metzger's EATOS (environmental assessment tool for organic syntheses) method and are compared with strictly analogous thermal processes. These include $\mathrm{C}-\mathrm{C}$ bond-forming reactions (arylation and alkylation) and selective oxidation and reduction reactions. In most cases, the photochemical method is experimentally simpler and less expensive than the thermal alternative. A disadvantage is that photochemical reactions are carried out in rather dilute solution, and this factor gives by far the main contribution to the assessment. However, if the solvent is recovered, the photochemical reaction is more environmentfriendly.
\end{abstract}

Keywords: green chemistry; photochemistry; $\mathrm{C}-\mathrm{C}$ bond formation; reduction, oxidation.

\section{INTRODUCTION}

The first unambiguous statement of the concept and the aim of green chemistry are more than 100 years old and refer to Giacomo Ciamician $[1,2]$. At the turn of the $19^{\text {th }}$ century, this great scientist reasoned that man was now able to prepare the compounds that nature made, and that the difference was not in the structure of such products, but in the way in which they were formed. Artificial synthesis in the laboratory was based on the use of harsh conditions, whereas the same compounds were formed in plants under (at least seemingly) much milder conditions. Ciamician surmised that the difference may lay in the fact that plants made use of solar light and set out to explore whether man could mimic nature also under this aspect and find a better chemistry in photochemistry.

Indeed, the above statement is taken from the introductory words of a presentation to the general assembly of the French Chemical Society in 1908 [1], where Ciamician illustrated the results of 10 years work on photochemical reaction, during which he had discovered several important classes of reactions.

\section{PHOTOCHEMISTRY AND GREEN CHEMISTRY}

Ciamician's work was certainly appreciated at the time, but was not further developed.

Synthetic photochemistry took new impetus since the 1950s, when many reactions were discovered (or, as it happened, rediscovered), so that now a large palette of photochemical transformations is available to the synthetic community and information is readily available, e.g., through books devoted

*Pure Appl. Chem. 79, 1831-2100. An issue of reviews and research papers based on lectures presented at the $1^{\text {st }}$ International IUPAC Conference on Green-Sustainable Chemistry, held in Dresden, Germany, 10-15 September 2006.

¥Corresponding author: E-mail: angelo.albini@unipv.it 
to this subject [3-5]. Actually, photochemical key steps are often introduced in many laboratories in synthetic schemes, although not as often as one may expect on the basis of the potential that photochemistry has.

Indeed, photochemical reactions are in fact a "green" alternative to thermal processes, as Ciamician had hoped. Certainly, as for the mildness of conditions, there is no comparison between photochemical and most thermal reactions, and undoubtedly the photon, differently from other activating agents or catalysts, leaves no residue after its action. Furthermore, from the point of view of atom economy, photochemical reactions are often advantageous because they cause deep-seated molecular transformations, so that often one obtains directly what would otherwise require several steps. Thus, the photon is the green reagent par excellence, or at least this is what photochemistry practitioners think. In order that this concept is generally accepted, suitable tests of its viability must be made $[2,6]$.

In other words, one must confront the question of where and when the application of photochemistry to organic synthesis is really advantageous from the environmental point of view, and then of course one must check whether this is a sensible choice of an economically sound procedure. The same criterion obviously applies also to nonphotochemical syntheses. In most cases, the label "green" is presently attached to any "novel" synthetic procedure, e.g., to a catalytic reaction that replaces a traditional procedure occurring under strongly basic or acid conditions, independent from the fact that the "catalyst" may be used in a relatively large amount, or may require several cocatalysts, be labile, toxic, expensive, and so on. In most instances, such reactions could be classed as innovative or unconventional methods, but they hardly comply with the requisites of green reactions. The fact that other classes of reactions share this shortcoming does not make it less desirable that photochemical reactions are examined from this point of view, in order to understand whether these are really green and whether there is any stumbling block (e.g., the price that justifies their limited use in synthesis).

Any green process must conform to the principle of atom economy [7-10], but the mere consideration of the stoichiometric equation Reagent $\rightarrow$ Product is not sufficient, and of the materials used, including the solvent, the auxiliary materials for the reaction and work-up must be considered. Actually, the examination should be extended to the precursors of the reagents used in order that the environmental potential is evaluated as completely as possible and, if this is the case, alternative syntheses are compared [7c]. Various methods have been proposed in order to take into account such factors [11-14]. In particular, Sheldon has proposed the use of the environmental quotient $E Q$, where $E$ is the amount of waste produced per unit amount of the desired product and $Q$ represents how environmentally undesirable such waste is, so that the $E Q$ quantity expresses the environmental burden caused by that process [12]. Clearly, such evaluation requires that the various environmental aspects of the process are evaluated.

A convenient tool for such analysis has been developed by Eissen and Metzger through the EATOS (environmental assessment tool for organic syntheses) procedure [14]. In this case, four indexes are evaluated. Two of these refer to the amount of chemicals involved:

- $\quad$ the mass index, $S^{-1}$, i.e., the sum of the raw materials used $(\mathrm{kg})$ per $\mathrm{kg}$ of the desired product;

- $\quad$ the environmental factor, $E$, i.e., the sum of the waste $(\mathrm{kg})$ per $\mathrm{kg}$ of product;

and two refer to the seriousness of their environmental impact, both for the feed and for the waste, by multiplying the above amounts by a factor $Q$. For the feed, $Q_{\text {input }}$ takes into account the environmental and social cost involved in its production, for the waste, $Q_{\text {output }}$ is an index of the potential damage to the ecosystem.

- $\quad E I_{\text {in }}=Q_{\text {input }} \cdot S^{-1}$;

- $\quad E I_{\text {out }}=Q_{\text {output }} \cdot E$.

In the method, the contribution of each main entry (reagents, solvents, catalysts, and auxiliaries) is evaluated separately for each index. Thus, high $E I_{\text {in }}$ values indicate wasteful processes in terms of 
consumed resources, high $E I_{\text {out }}$ process of high environmental impact. Further particulars and the significance of the method are discussed in the original publication [14]. As a further element for the assessment, the price per $\mathrm{kg}$ of product has been calculated.

We used this method for assessing the "greenness" of some exemplary photochemical reactions vs. recently reported thermal alternatives. It was deemed appropriate to refer to lab scale, both because most "innovative" reactions have been developed only in a small scale and because the number of photoreactions presently carried out in the industry, although quite limited, is sufficient to demonstrate that scaling up is no insurmountable barrier for such reactions and a variety of technical expedients have been devised for different cases. Indeed, several photochemical processes have been considered advantageous in the industry, and a satisfactory solution for carrying them out in the desired scale has been found $[15,16]$. Furthermore, it even appears that solar light can be used for some useful chemical reactions $[17,18]$. In the present work, literature data most often referred to the $0.1-1 \mathrm{~g}$ scale have been compared and conventionally referred to the $1 \mathrm{~kg}$ (of the product) scale.

\section{ALKYLATION BY USING ALKANES}

Forming radicals from alkanes would seem to be one of the simplest ways of functionalizing a $\mathrm{C}-\mathrm{H}$ bond. Starting from a symmetric reagent (e.g., a cycloalkane), controlled pyrolysis may be a viable method. On the other hand, hydrogen abstraction may be carried out in the cold by using a photocatalyst, such as the decatungstate anion. In the presence of an electrophilic alkene, the radical is trapped and subsequent back electron transfer and protonation yield the alkylated product and regenerate the photocatalyst. In this way, methyl cyclohexylpropanoate has been prepared from the cyclohexane and methyl acrylate (Scheme 1a; this is a $100 \%$ atom economy process, all the atoms of the reagents are incorporated into the products) in acetonitrile in $75 \%$ yield [19].

a)

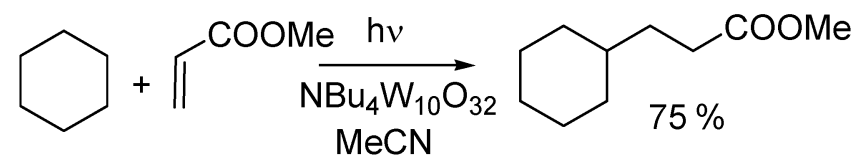

b)

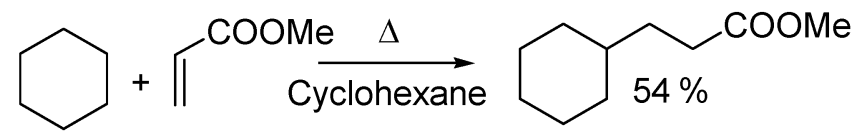

Scheme 1 Photocatalyzed (a) and thermal (b) alkylation of methyl acrylate.

As in Fig. 1, the contribution of the reagents to the value of $S^{-1}$ and $E$ depends on the fact that an excess cyclohexane is used (recovery has not been considered at this stage; likewise, the catalyst has been considered as used up). $Q$ takes into account the potential environmental impact of the chemicals involved, through such elements as the R-phrases and the LD values. In this case, the value of $E I_{\text {in }}$ depends on the use of a toxic reagent such as the acrylate, which is consumed in the reaction to give less toxic products, hence the lower value of $E I_{\text {out }}$.

However, the major contribution to all of the indexes is given by the solvent ( $>90 \%$ in all the cases). This is a characteristic of photochemical reactions. Dilute solutions are used because light must penetrate into the solution to a significant depth in order to make the molecules absorb and react, and too high an absorbance would limit absorption (remember the Lambert-Beer law) and reaction to the first thin layer close to the lamp (formation of a film due to decomposition and polymerization of the 


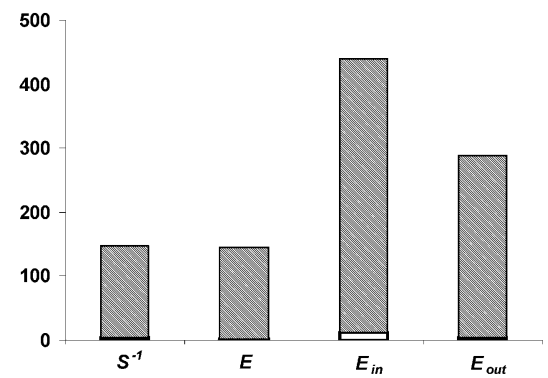

a)

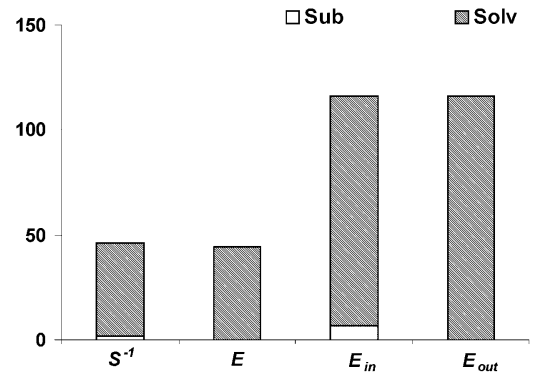

b)

Fig. 1 Assessment according to the EATOS method of the photocatalytic (a) vs. the thermal (b) synthesis of methyl cyclohexylpropanoate; reagents (Sub) and solvent (Solv) contribution to each index are indicated. The contribution by the catalyst is too small to be detected in Fig. 1a.

solute is often observed if this precaution is not observed). Furthermore, the solvent must be transparent to the wavelength range used and reasonably inactive. In this case, acetonitrile has been used, a convenient solvent for many photochemical reactions, but not an environmentally suitable choice (the reaction works also in neat cyclohexane, although it is quite slow in that case). In this preliminary assessment, the solvent is considered as used up. When planning application, a sensible evaluation of the management and recovery of the solvents is a key issue in photochemistry.

The same product can be obtained thermally by passing a mixture of the reagents through a stove at $400{ }^{\circ} \mathrm{C}$, with a residence time of $1 \mathrm{~min}$ ("ane" reaction) [20].

As shown in Fig. 1, the environmental performance of pyrolysis is better than that of the photochemical reaction and the various indexes follow similar patterns (cyclohexane is used in excess also in this case, and considered as the solvent). In fact, the energy required for heating the mixture at the required temperature is lower than that consumed by the low-pressure arc used for the irradiation. As a matter of fact, in both cases, the main contributions to the environmental impact (and by consequence to the price) are by far those due to the solvents (the work-up, which has been reported by column chromatography in both cases, has not been considered, since distillation could certainly be applied). Actually, also in the pyrolytic method more than $90 \%$ of the environmental impact is due to the use of excess cyclohexane. Both thermal and photochemical pathways offer a wide margin to improvement, should optimization of the process be considered.

We finally evaluated the price of production of the alkylated esters by the two methods. The economic assessment is obviously one of the main issues when discussing the feasibility of an industrial process. However, this requires that a thorough assessment of the current price of each reagent for a bulk purchase is done. Since this was not possible, we used the prices quoted in a specialty chemicals catalogue (Aldrich) and chose the largest amount listed. We hoped that this gives a relative assessment, even if the resulting prices were 10 to 100 times too high. For the above alkylation, these were $€ 1300 / \mathrm{kg}$ for photocatalysis and $€ 200 / \mathrm{kg}$ for thermolysis.

In summary, thermolysis is clearly preferable to photocatalysis for a very simple process such as radical alkylation. Notice, however, that introducing some complexity may easily lead to reversion of the result. As an example, the photocatalytic alkylation of acrylate is equally well accomplished by using an alcohol, e.g., iso-propanol [21], and involves selective abstraction of the hydrogen $\alpha$ to the $\mathrm{OH}$ group. In this case, there is no direct thermal alternative, since at a high temperature other processes compete and thermally activated initiators are not sufficiently selective for the desired bonds. Nonphotochemical alternatives must thus resort to a different approach. As an example, the $\alpha$-hydroxyl radical has been obtained from acetone by cathodic reduction, but addition to methyl acrylate gives a reasonable yield only with a large acetone excess and under microwave absorption, making the process certainly more expensive [22]. 


\section{ARYLATION BY ARYL HALIDES}

As is well known, aryl halides do not easily undergo substitution reactions. Both metal (usually Pd) catalysis and photolysis have opened new paths in these reactions. The two methods were compared, choosing in this case a more complex, and obviously more expensive, target molecule, the alkynybenzoxazole shown in Scheme 2. This can be prepared from the corresponding chloro (or bromo) benzoxazole either by Sonogashira coupling [23] or by irradiation [24].

a)

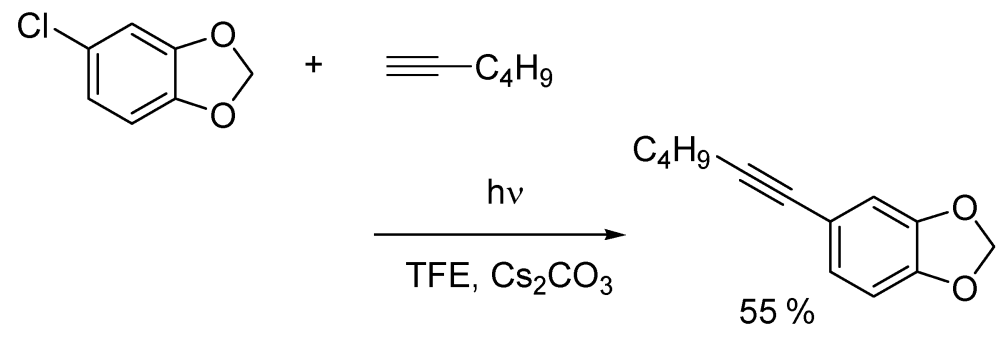

b)<smiles>Brc1ccc2c(c1)OC[Te+]2[Te]</smiles>

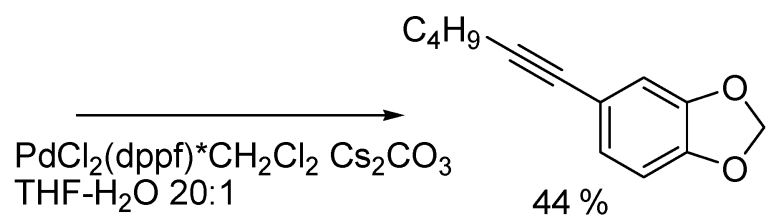

Scheme 2 Photochemical (a) and Pd-catalyzed (b) arylation of an alkyne.

The photochemical reaction has been carried out in the presence of the alkyne in trifluoroethanol containing cesium carbonate. The potential environmental impact is higher than in the previous cases, and the solvent contributes largely, as does the excess of hexyne used. The $E I_{\text {in }}$ value $\left(>E I_{\text {out }}\right)$ is strongly affected by the excess of the last reagent (quite volatile and flammable). However, a thermal reaction starting directly from the alkyne has not yet been accomplished. A related example via metal catalysis makes use of the potassium trifluoborate salt of the alkyne, not of the alkyne itself. The preparation of this reagent increases considerably the environmental burden. Thus, the comparison is more to the advantage of the photochemical alterative than appearing from the diagram above. The preparation of the salt has been taken into account when evaluating the price of the thermal synthesis, of which it constitutes the main single contribution.

As for the last point, the price reaches the enormous figure of $€ 35000 / \mathrm{kg}$, in comparison to $€ 18000 / \mathrm{kg}$ for the photochemical reaction. Even when considering the previous caveat about the much lower prices obtained by industries, these data make it apparent how expensive are most syntheses as published in scientific journals. In such papers, the aim is exploring the scope of some (new) reaction, with no effort toward optimization or ascertaining the economic viability. In this case, even recovery of the solvent would lower only in part the price of the product.

To summarize, carrying out an unconventional synthesis such as that of the alkynylbenzoxazole considered here is quite expensive, mainly because of the high price of the reagents used, although the 


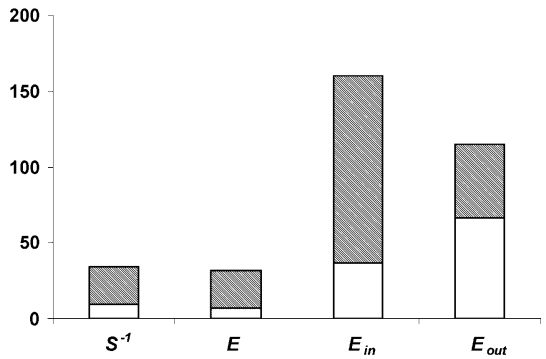

a)

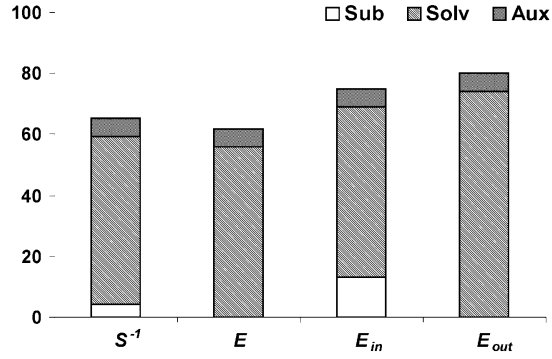

b)

Fig. 2 Assessment according to the EATOS method of the photochemical (a) vs. the thermal (b) synthesis of an alkynylbenzoxazole.

prices vary over a large span when using different methods. The photochemical reaction is in fact about as polluting as the Pd-catalyzed alkylation, but the price is only a fraction. By using the EATOS method, the performance of the photochemical reaction has been assessed for some further arylations of this type, and found to be generally better than that of Pd-catalyzed reactions (Fig. 2).

\section{OXIDATION}

Oxygenations, in particular those via singlet oxygen, are one of the few classes of reactions where the photochemical method is recognized as the most convenient one and actually is routinely used in the industry, for instance, for the synthesis of some fragrances [25]. Thus, this point has been considered as assessed and a different reaction has been considered here, viz. the selective oxidation of benzyl alcohols or of methylbenzenes to aldehydes by using molecular oxygen (Scheme 3). For the aromatic derivatives, this can be carried out by using molecular oxygen either upon Pd catalysis [26a] or upon acridinium photosensitization [26b].

a)<smiles>Cc1ccc(C)cc1</smiles>

b)<smiles>Cc1ccc(CO)cc1</smiles>

Scheme 3 Oxidation of an alcohol to the aldehyde by using molecular oxygen either under Pd catalysis (a) or by photosensitization (b).

As it appears from Fig. 3, the environmental indexes are rather high due to the use of chemicals that have a strong impact. The photochemical reaction as taken from the literature suffers from the overwhelming contribution of chloroform used as the solvent, which precludes the adoption of the method. Other solvents that are environmentally less demanding (e.g., acetonitrile in this electron-transfer photooxidation) or at least not much worse, and in that case the assessment would give a more reason- 
able result. Notice that even when carried out in chloroform as reported in the literature, the price of the photochemical alternative is the lower one ( $€ 1500$ vs. $10300 / \mathrm{kg}$ ). Furthermore, when considering the contribution due to substrate and auxiliary materials and not that of the solvent, the $E I_{\text {in }}$ index becomes favorable over the photochemical path (2.2 vs. 4.2).

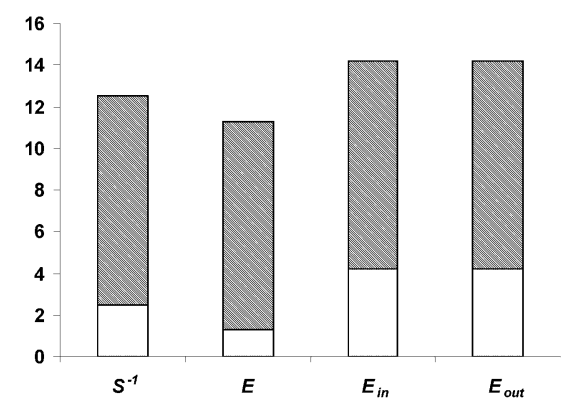

a)

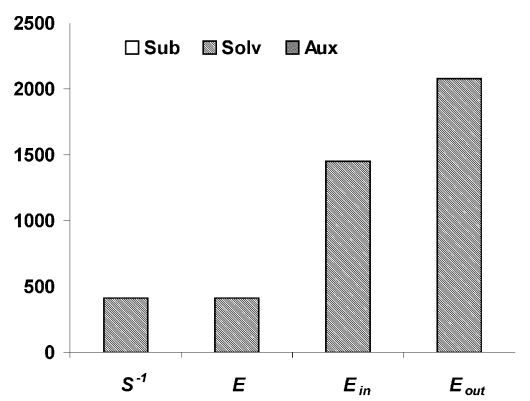

b)

Fig. 3 Assessment according to the EATOS method of the selective alcohol oxidation under photosensitized (a) vs. thermal (b) conditions. The contribution by the substrate is too small to be appreciated in (a).

\section{REDUCTION}

As mentioned above, photochemical oxidations are rather commonly used. However, photoreduction may play an important role as well. As an example, the regioselective reductive opening of the threemembered ring in the ketoepoxide shown in Scheme 4 can be carried out by using a strongly reducing agent, such as in situ prepared $\mathrm{Ti}^{\mathrm{III}}$ [27] or sodium hydrogen telluride [28]. However, the same process can be carried out under mild conditions provided that the reaction occurs via the excited state, much easier to reduce than the corresponding ground state. In fact, the excited state is reduced by weak electron donors, such as aliphatic amines [29] or benzimidazolines [30]. The EATOS analysis carried out on the thermal process with $\mathrm{Ti} i \mathrm{II}$ and on the $\mathrm{hv} / \mathrm{NEt}_{3}$ systems shows that the main contributions are essentially due to the reagents in the first method and to the solvent in the latter one; indeed, the indexes for the photoprocess are higher, but only because of the solvent ( $>95 \%$, see Fig. 4). As for the price, this is estimated as $€ 2700$ in the photochemical and $€ 99900 / \mathrm{kg}$ for the thermal process, where quite expensive reagents were used. Again, not considering the solvent contribution, the $E I_{\text {in }}$ index is favorable for the case of the photoreaction (26 vs. 126).

a)<smiles>CCN(C)C(=O)C(=O)c1ccccc1</smiles>

b)<smiles>O=C([C+]=COc1ccccc1)C(O)CC(O)C(=O)c1ccccc1</smiles>

Scheme 4 Regioselective reductive opening of an epoxide by using irradiation in the presence of triethylamine (a) and by using in situ prepared $\mathrm{Ti}^{\mathrm{III}}$ (b). 


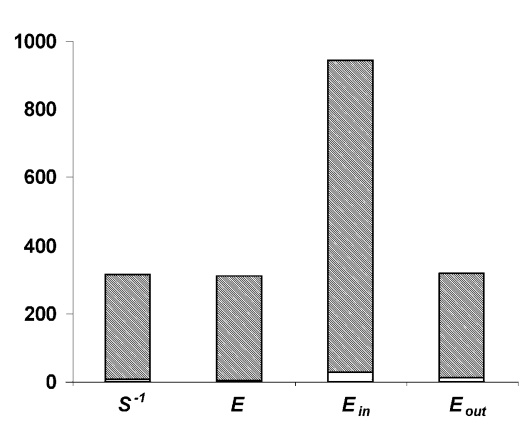

a)

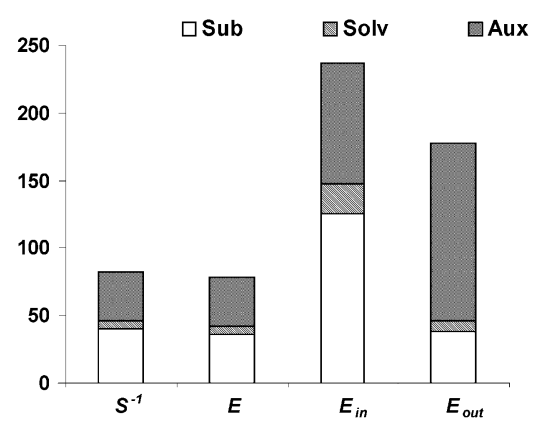

b)

Fig. 4 Assessment according to the EATOS method of the photochemical (a) vs. the thermal (b) preparation of a $\beta$-hydroxyl ketone from the $\alpha, \beta$-epoxyketone. The contribution by the substrate can be barely appreciated in (a).

\section{CONCLUSIONS}

The above analysis through the EATOS protocol that takes into account the environmental potential of a chemical reaction broken down according to the various contributions is evidence of positive and negative aspects of photochemical reactions. These reactions generally show a good environmental performance, supporting the fact that photochemistry is indeed a sustainable alternative. In the examples discussed above, the electronically excited state reacts, and since it lies quite high in energy (60-150 $\mathrm{kcal} / \mathrm{mol}$ ), reactions that would be prohibited in the ground state are possible, indeed under exceptionally mild conditions.

Many reactions of the excited states have no ground-state counterpart. In other cases, there is a parallelism because the excited state cleaves giving a highly reactive intermediate that may be generated also thermally, but then either starting from more elaborated reagents and/or under more severe conditions (examples of an alkyl radical, a phenyl cation, a radical cation, or anion are among the reactions reported above). Apart from the contribution by the solvent, the environmental performance is better because no additive is required and usually less elaborate conditions are involved with respect to thermal reactions where a chemical reagent or a catalyst is used.

If the solvent is considered and it is assumed that it is discarded after use, as in the above assessments, the photochemical reactions give a bad environmental performance, although they remain less expensive. Obviously, if industrial application is seriously considered, a sensible choice and management of the solvent is the first issue to be confronted. This is an important limitation, since recycling does not prevent expenses relative to safe handling, recovery, and storage. However, once this limitation is overcome, the potential of photochemistry for sustainable synthesis becomes apparent and the role of photochemistry as a green method can be appreciated. The only case where the thermal alternative performs better is that involving a simple, noncatalyzed thermolysis to give directly the desired intermediate, a reaction course that is not that common, unfortunately.

For reactions on a small scale, such as those considered here, the environmental assessment has little significance, since in this case the price of manpower largely exceeds that of chemicals and of any technical expenses. The assessment makes sense only when an industrial application is considered, and in that case it is important to distinguish as early as possible whether a synthetic method can be considered. The above discussion at least indicates that the photochemical method should not be a priori discarded. This is experimentally quite simple and does not produce waste from activators or catalyst. Most importantly, in several cases it starts from less functionalized reagents, and skipping a step obviously involves a strong decrease of the environmental impact, as quantitatively demonstrated in other cases [31]. 
The relatively limited use of photochemical reactions in industrial applications is possibly due to incomplete knowledge of this discipline, sometimes partially covered in university courses, rather than to any practical limitation in carrying out them. Clearly, a starting investment is required since an ad hoc apparatus has to be built, and the expertise on scaling up a photochemical process is certainly much less widespread than for thermal reactions. However, the unnegligible number of industrial photochemical reactions presently carried out around the world testifies to the viability of this method. A larger use of photochemistry in explorative studies in R\&D (and university) laboratories would reasonably lead to a more extensive application in industrial processes, with undoubted advantage from the environmental point of view.*

\section{REFERENCES}

1. (a) G. Ciamician. Bull. Soc. Chim. Fr. [4] 3, i (1908); (b) G. Ciamician. Science 36, 385 (1912).

2. A. Albini, M. Fagnoni. Green Chem. 6, 1 (2004).

3. J. Mattay, A. Griesbeck (Eds.). Photochemical Key Steps in Organic Synthesis, VCH, Weinheim (1994).

4. A. G. Griesbeck, J. Mattay (Eds.). Synthetic Organic Photochemistry, Marcel Dekker, New York (2005).

5. W. Horspool, F. Lenci (Eds.). CRC Handbook of Organic Photochemistry and Photobiology, $2^{\text {nd }}$ ed., CRC Press, Boca Raton, FL (2004).

6. A. Albini, M. Fagnoni, M. Mella. Pure Appl. Chem. 72, 1321 (2001).

7. (a) B. M. Trost. Science 251, 1471 (1991); (b) R. A. Sheldon. Pure Appl. Chem. 72, 1233 (2001); (c) M. Eissen, R. Mazur, H. G. Quebbemann, K. H. Pennemann. Helv. Chim. Acta 87, 424 (2004).

8. P. T. Anastas, J. C. Warner. Green Chemistry: Theory and Practice, Oxford University Press, Oxford (1998).

9. P. Tundo, P. T. Anastas, D. StC. Black, J. Breen, T. Collins, S. Memoli, J. Miyamoto, M. Polyakoff, W. Tumas. Pure Appl. Chem. 72, 1207 (2000).

10. (a) T. Collins. Science 291, 48 (2001); (b) W. Leitner. Science 284, 1780 (1999).

11. N. Winterton. Green Chem. 3, G73 (2001).

12. R. A. Sheldon. CHEMTECH 3, 38 (1994).

13. E. Heinzle, D. Weirich, F. Brogli, V. H. Hoffmann, G. Koller, M. A. Verduyn, K. Hungerbühler. Ind. Eng. Chem. Res. 37, 3395 (1998).

14. (a) M. Eissen, J. O. Metzger. Chem. Eur. J. 8, 3580 (2002); (b) M. Eissen, J. O. Metzger. EATOS, Environmental Assessment Tool for Organic Syntheses, <http://www.chemie.uni-oldenburg.de/ oc/metzger/eatos/>.

15. (a) A. M. Braun, M. T. Maurette, E. Oliveros. Photochemical Technology, John Wiley, Chichester (1991); (b) H. D. Scharf. Angew. Chem., Int. Ed. Engl. 33, 2009 (1994).

16. (a) P. Hulme, P. E. Turner. Chem. Process Eng. 48, 96 (1967); (b) Y. Ito, S. Matsuda. Annu. N. Y. Acad. Sci. 147, 618 (1969).

17. (a) K. H. Funken, F. J. Müller, J. Ortner, K. J. Riffelmann, C. Sattler. Energy (Oxford) 24, 681 (1999); (b) N. Monnerie, J. Ortner. J. Sol. Energy Eng. 123, 171 (2001).

18. (a) M. Oelgemöller, N. Healy, L. de Oliveira, C. Jung, J. Mattay. Green Chem. 8, 831 (2006); (b) J. Mattay. Chem. Unserer Zeit 36, 98 (2002).

19. D. Dondi, M. Fagnoni, A. Molinari, A. Maldotti, A. Albini. Chem. Eur. J. 10, 142 (2004).

20. J. O. Metzger, K. Klenke, J. Hartmanns, D. Eisen. Chem. Ber. 119, 508 (1986).

21. D. Dondi, M. Fagnoni, A. Albini. Chem. Eur. J. 12, 4153 (2006).

22. M. Atobe, S. Fujiwara, T. Nonaka. Bull. Chem. Soc. Jpn. 75, 123 (2002).

*The EATOS project files for the above reactions are available from the authors upon request.

(C) 2007 IUPAC, Pure and Applied Chemistry 79, 1929-1938 
23. G. A. Molander, B. W. Katona, F. Machrouhi. J. Org. Chem. 67, 8416 (2002).

24. S. Protti, M. Fagnoni, A. Albini. Angew. Chem., Int. Ed. 44, 5675 (2005).

25. W. Rojan, H. U. Warnecke. Dragoco Rep. 27, 157 (1980).

26. (a) K. P. Peterson, R. C. Larock. J. Org. Chem. 63, 3185 (1998); (b) K. Ohkubo, K. Suga, K. Morikawa, S. Fukuzumi. J. Am. Chem. Soc. 125, 12850 (2003).

27. C. Hardouin, F. Chevallier, B. Rousseau, E. Doris. J. Org. Chem. 66, 1046 (2003).

28. A. Osuka, K. Takaoka, H. Suzuki. Chem. Lett. 271 (1984).

29. J. Cossy, A. Bouzide, S. Ibhi, P. Aclinon. Tetrahedron 47, 7775 (1991).

30. E. Hasegawa, N. Chiba, A. Nakajima, K. Suzuki, A. Yoneoka, K. Iwayma. Synthesis 1248 (2001).

31. H. U. Blaser, M. Eissen, P. F. Fauquex, K. Hungerbühler, E. Schmidt, G. Sedelmeier, M. Studer. In Large-Scale Asymmetric Catalysis, H.-U. Blaser, E. Schmidt (Eds.), pp. 91-104, Wiley-VCH, Weinheim (2003). 